\title{
SEM/EDS as a Tool to Investigate Pyrolysis Induced Transformations in Chicken Feather Fibers
}

\author{
Zaheeruddin Mohammed $^{1}$, Shaik Jeelani ${ }^{1}$ and Vijaya Rangari ${ }^{1 *}$ \\ ${ }^{1 .}$ Department of Material Science and Engineering, Tuskegee University, Tuskegee, USA. \\ * Corresponding author: vrangari@tuskegee.edu
}

Scanning Electron Microscopy (SEM) is a dedicated field of science in which electron microscope uses the beam of electrons which is scanned in a raster pattern over the sample surface allowing imaging and quantification of surface topographic features. In conjunction to SEM, Energy Dispersive Spectroscopy (EDS) can be used to characterize the chemical composition of materials utilizing the electrons ejected from atoms comprising the samples surface.

Chicken feather fibers (CFF) which are abundant source of keratin fiber $(92 \%)$ can be explored for fabrication of materials useful for applications like storage, separation and electronics. Biochar obtained from the pyrolysis of biomasses have shown to be very promising alternatives to high cost carbon fillers to improve mechanical, electrical and thermal properties [1]. However, pyrolysis of protein based materials can be complex due to the presence of more varied amino acids in different sequences and mole ratios. CFF structure consists of semi crystalline and crystalline regions, which makes the thermal decomposition very intricate due to its tendency to melt below melting transition temperatures and inter and intramolecular crosslinking [2].

The aim of the present work is to implement the combination of SEM/EDS (Jeol JSM - 7200 F) techniques to investigate pyrolysis induced physical and chemical transformations in chicken feather fibers. Thermal treatment using Thermo gravimetric analysis (TGA) under constant flow of nitrogen $(50 \mathrm{~mL} / \mathrm{min})$ was used to pyrolyze the CFF. Feather fibers were heated from 30 to $200{ }^{\circ} \mathrm{C}$, then they were treated isothermally for 2 hours at $200{ }^{\circ} \mathrm{C}$ and then heated up to $400{ }^{\circ} \mathrm{C}$ at the rate of $10{ }^{\circ} \mathrm{C} / \mathrm{min}$. Isothermal heating was done to promote crosslinking of bonds by reaction of amine-carboxyl side chain groups during pyrolysis [3]. Pyrolyzed chicken feather fibers were then investigated via SEM for structural morphology and quantitative estimation of elemental atomic percent (At \%) using EDS was done by implementing ZAF method.

From SEM micrographs obtained it can be observed that the fibrous structure of the feather was retained after pyrolysis. The borders of each individual fiber was still intact and distinguishable because of stabilization mechanism, possibly due to intermolecular crosslinking such as isopeptide bond formation. However, there was change in color from light brown to black. It was also observed that the long barbules present on the side of barbs were decomposed due to thermal treatment. (Fig 1) Quantitative analysis of EDS studies of fibrous section of feathers (Fig 2) revealed that there was decrease in at \% of Sulphur from $2.56 \%$ to $0.58 \%$ which is due to disulfide bond cleavage. There was also slight reduction in the At \% of Oxygen and Nitrogen indicating $\mathrm{H}_{2} \mathrm{O}$ emission and cleavage of $\mathrm{HN}-\mathrm{C}$ bond respectively. As a result of decrease in other constituent elements At \% of carbon increased from $60.95 \%$ to $66.72 \%$, indicating there was no loss in carbon content of chicken feather fiber after pyrolysis (P-CFF).

The knowledge acquired through SEM/EDS studies confirms that isothermal treatment during pyrolysis of CFF helps in retaining fibrous keratin which can eventually produce microporous material with large 
surface area at higher temperatures. The carbon thus synthesized can be a useful precursor for storage, separation, purification and reinforcing applications.

References:

[1] F Qin and C Brosseau, Journal of Applied Physics 111.6 (2012), p. 4.

[2] E Senoz et al., Polymer Degradation and Stability 97.3 (2012), p. 297.

[3] E Senoz and RP Wool, Journal of Applied Polymer Science 118.3 (2010), p. 1752.
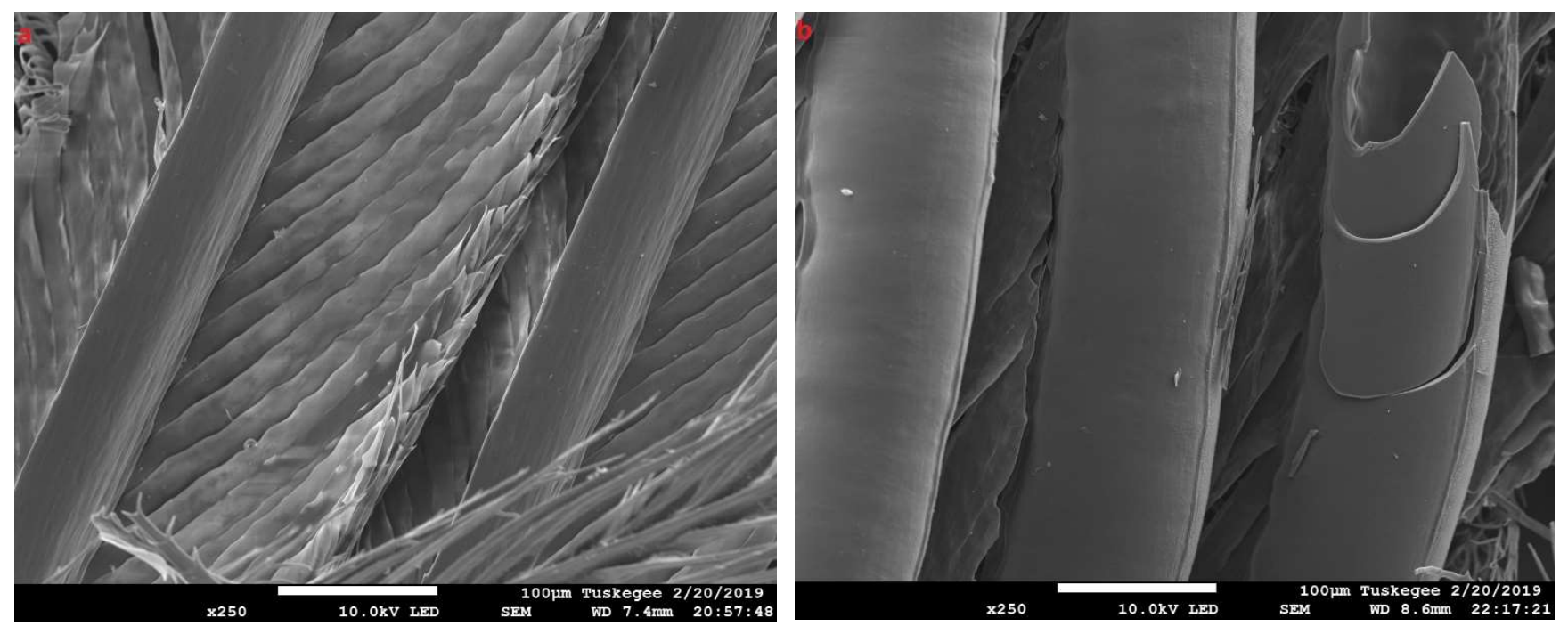

Figure 1. SEM micrographs of a) CFF and b) PCFF

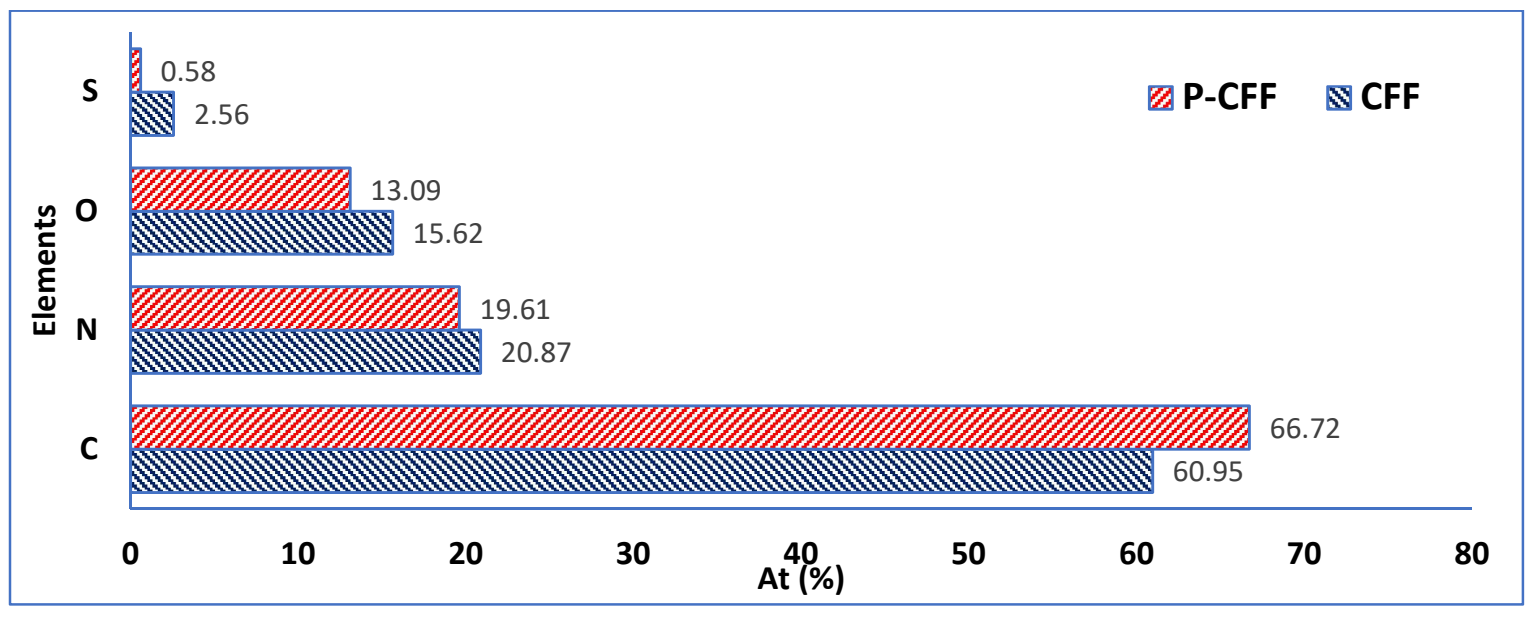

Figure 2. Major elemental constituents of chicken feather fibers before and after pyrolysis. 